\title{
Blue Light Therapy and the Circadian Rhythm: Effect on Southern California High School Students
}

\author{
Aaron Hoy ${ }^{1}$ \\ ${ }^{1}$ Long Beach Polytechnic High School, Long Beach, CA, USA
}

\section{ABSTRACT}

Blue-light therapy is well known for curing patients with Seasonal Affective Disorder (SAD), where the patient experiences depressive factors. In this study I used blue-light therapy in the form of blue-light lightboxes from the company Circadian Optics, as seen on shark tank. Instead of using the lightboxes to cure SAD, they were instead used to see if they provoked the participants sleep quality. The participants from Polytechnic high school were selected from the Badminton Team, where the athletes woke up at five a.m. on school days. This ensured that the crowd was overall tired and fatigued. Over the four-week testing course, participants proved positive results.

\section{Introduction}

Currently in the United States, an estimated 15.3 million students are in high school grades 9-12 (NCED 2019). High school sets up the student for college and life outside of school which can become time consuming and stressful. With the rising amount of workload and learning material, students are challenged and as a result are losing sleep, affecting focus the following day, therefore weakening education and grading conduct. The condition of not having enough sleep is known as sleep deprivation (Ranasinghe et al, 2018). Sleep deprivation can be related to "school responsibilities, family commitments, after-school jobs, extracurricular activities, and social media" illustrating the busy lives high-school students pursue (Jackson et al, 2020). This impacts personal lives heavily and balance can be difficult.

The increasing amount of sleep deprivation is not only unhealthy but affects students' performance in school due to morning drowsiness which continues throughout the day. It is widely believed that students manage personal lives and school effectively and receive the vital amount of sleep to get through the day, but that is not the case for the majority. Juggling personal ties, whether it be watching siblings or managing a part-time job in addition to schoolwork including studying, homework, projects, etc. is heavily stressful on a student. A high-school experiment concluded that "more than 1 in 4 students (26\%) get 5 hours or less of sleep" capturing the lack of slumber students should be receiving which is 8-10 hours (Jackson et al, 2020). Since students' brains and bodies are still developing during the teenage years during high school, the lack of sleep affects brain development and health, which is concerning. Lack of sleep is associated with "an increased risk for injuries, hypertension, obesity and depression" which can harshly impact a student's lifestyle and mental health (Jackson et al, 2020). The loss of sleep causes a slowing of brain energy in the frontal cortex, resulting in higher anxiety, a shorter attention span, and a miserable mood (Ranasinghe et al, 2018). These factors not only impact the individual, but also their work ethic which could further damage the student's mental state and student life. Although schedules are intangible and vary from one student to the next, blue-light provides a promising tool for high-school students' hectic lives as it can improve sleep quality, energy levels, and attentiveness. 


\section{Literature Review}

According to a university study conducted by Pradhan, adolescents require more sleep than adults and pre-adolescent children, but this is limited by schoolwork obstacles and early wake-up times (2017). Sleep deprivation can affect the brain, by limiting alertness, and the body, by increasing the production of insulin which can lead to further issues. The constant loss of sleep produces cortisol, which makes the human cells more resistant to insulin. Insulin is the hormone that regulates the amount of glucose within the person's blood. If insulin resistance increases due to cortisol from lack of sleep, the body becomes more susceptible to obesity. This only opens up more negative pathways that sleep deprivation can lead to for a student. The sleep cycle is controlled by the function named the circadian rhythm, which is the human body's personal clock which decides when to stay awake or go to sleep (Loredan-Sabina et al. 2019). Moreover, the circadian rhythm functions off blue-light which is present in different amounts in all forms of light, but most notably in electronic devices. Blue-light sends the circadian rhythm a signal to keep the user awake, no matter what time. The natural hormone melatonin, which sends the brain a signal to sleep (and wake up), stops when exposed to blue-light, so if blue-light is used at inappropriate times, the body cannot fall asleep. This hormone is released approximately two hours before the person's normal bedtime, and if blue-light is exposed highly during this time they cannot fall asleep. The increasing use of electronic devices in school affects students mainly, due to late night usage causing students to have trouble falling asleep. All in all, the major lack of sleep, in addition to personal workload, causes students to feel drowsy and non-attentive at school known as morning fatigue. Improvements and changes to student workload remain ignored, therefore scarce resolutions exist. Sleep is the major factor that can be improved to decrease morning fatigue and increase alertness.

\section{Blue-light Therapy: Past and Present}

Although blue-light may be viewed as harmful (as in electronics mainly) for its effect on sleep, light has been shed on the positive side of blue-light with a promising change in the therapy field (Miura 2018). If blue-light is used at appropriate times, it can be a useful tool.

Over the years, therapeutic devices have come and gone, but one of the uprising forms of therapy has been used in the form of a blue-light box which is ultimately harmless (van Hoof et al. 2012) and can only produce positive results. Blue-light boxes most notably used as therapy for a disorder known as Seasonal Affective Disorder (SAD) which shows promising results based on the circadian rhythm. Furthermore, blue-light lightboxes were designed to cure the brain psychologically/mentally for a certain amount of time with SAD patients (Meesters et al. 2016). The positive effects of using the lightbox in the study included mainly curing depression, higher energy levels, better work ethic, and an easier time falling asleep; negative effects were non-existent. As of now, blue-light boxes have been seen on Shark Tank from the company Circadian Optics (the company I used during testing). Regarding the proposal, the company advertised the boxes to maintain a healthy sleep cycle, improve mood, help concentration, and improve energy. Circadian Optics is a successful company where the CEO and co-founder, Amber Leong, received Lori Greiner and Mark Cuban's investments. The company is up-and-coming within the world of blue-light therapy.

Other pre-existing forms of blue-light therapy usage include various forms of phototherapy acne treatment, sleep disorder treatments, etc. Acne treatment with blue-light physically touches the skin with rays, but is not damaging or harmful, which proves blue-light to be safe (Bagherani 2016). Blue light irradiation is effective in acne treatment proven within a study, where patients were gathered with symmetrical acne on the face. Only one side of the face was treated with blue-light, the results positively cured the tested side in comparison to the non-tested side (Tien-Yi Tzung et al. 2004). Another blue-light device study concluded "blue-light LED therapy significantly improved the outcome of mild to moderate inflammatory acne lesions...faster resolution and overall improvement in patients' skin condition and faster resolution of the lesions" demonstrating how blue-light is successful for acne deplenishing (Gold et al. 2011). On the other hand, Blue-light therapy for sleep apnea has shown relief in recent years as it assuages pain and 
relieves headaches allowing for better sleep (Miyamoto et al. 2003). LEDs which emit blue light allows the brain to stimulate happiness and digress depression

further proving blue light as a remedy (Desan et al. 2007). Three other types of sleep disorders are also treated with blue light in forms of lamps, special dawn room lights, and visors. The

disorders include Delayed Sleep Phase Syndrome (DSPS), Advanced Sleep Phase Syndrome (ASPS), and Insomnia which is not necessarily a disorder but in close relation to it. DSPS causes the person to go to sleep two or more hours later than the average human and wake up two or more hours later than everyone. ASPS is the opposite where the person goes to sleep two or more hours before the average human and wakes up earlier. Insomnia is simply where a person constantly has trouble falling asleep. All can be fixed with blue-light therapy where the light fixes the person's circadian rhythm or the "clock of the human body." This proves that blue light can be a universal resolution to various issues regarding the body and sleep cycle.

\section{Blue-Light as an Invasive Method for Solving Morning Fatigue}

With the known benefits of using blue light as therapy, it has yet to be applied to students in high school exposing a gap in research. Blue-light from natural light in the morning proves to be effective in advancing the melatonin production allowing one to be more wide awake (Lack et al. 2007). Therefore, if executed correctly, utilizing a lightbox originally designed for individuals with sleep disorders, and applying it to high school students instead who suffer heavy workload and early wake-up times may improve work ethic and overall provide a healthier lifestyle. I will utilize the benefits described by the Circadian Optics's lightboxes to see the effect on high-school students with bustling schedules and routines. Possibly improving alertness, which would improve absorption of information, and making it easier to fall asleep/have a deeper sleep, blue light boxes applied to adolescents in high school is an invasive method that I will explore with my research.

\section{Hypothesis}

Multiple remedies for humans have been experimented with for sleep and energy, but my research will be focusing on utilizing a blue-light lightbox in efforts to solve high school students' morning fatigue, improve work ethics, and sleep. Using the existing knowledge of blue-light and its pros and cons, I will test for all the factors sleep affects. This brings me to my research question: "Does the use of blue-light therapy positively impact current high-school students in Southern California regarding morning fatigue and work ethic?"

\section{Method}

The research project data collection took place over the course of five weeks in order to test my hypothesis. The first week was dedicated to collecting participants and a control group who did not participate in the testing, and two weeks per testing session. Various surveys were conducted via Google Forms during the research period including: a presurvey, daily log, and post-survey. Four lightboxes were used in this experiment from Circadian Optics' the "lumine lamp" containing 10,000 lux which is the ideal power for blue light to react with the human circadian rhythm. All data was charted in the following sections according to topic/subheading.

\section{Week One | Control Group and Participant Collection}

The first week was projected to gain a control group to answer an identical pre-survey that the 16 testing participants also filled out. The control group consisted of ten voluntary students from Long Beach Polytechnic High School, 912th grade. Data from the control group 
was needed to compare to the data from the 16 participants. 16 participants for the testing were collected by issuing parental consent forms. Participants were gathered specifically from the Polytechnic High School Badminton team (since I had easy access to the team as I am a member myself, also the team wakes up at four a.m. because practice is a zero period) presenting a tight schedule that students follow, perfect to test for the effects of blue-light. All 16 then took a pre-survey which asked questions regarding current sleep and work ethic levels. These pre-survey questions were to collect data of current sleep schedules and attentiveness in school (chart seen in the results section). After all participants were solidified and the pre-surveys were completed, the 16 participants were divided into two groups for two different sessions, therefore 8 participants for each session.

\section{Week Two-Five | Procedure}

Both sessions were over the course of two weeks (weekdays only) totaling four weeks. Each was conducted with the use of four lightboxes, a pair of participants per lightbox, for a total of eight people in one session. Each pair sat in front of the lightbox (about 10-12 inches away) for 15 minutes doing as they wished, not having to physically look at the box, just be physically in front of it without faces covered in any way. Each day, the participants filled out a short log recapping the previous day containing questions regarding sleep quality and drowsiness at school. The log questions were all based on a 1-5 ranking system, five being the highest/positive option, and one being the lowest/negative option. The daily $\log$ was conducted every day for 10 days each session, equaling 20 days. At the end of the two-week session, a post-survey was conducted. It asked about the overall experience using the lightbox over the past ten days, asking the participant to rank on a scale of 1-5 certain aspects: lightbox effectiveness and personal experience/results. After the post-survey was completed for both sessions, all data was charted by the Google sheets conversion option available by Google forms.

\section{Testing Setup}
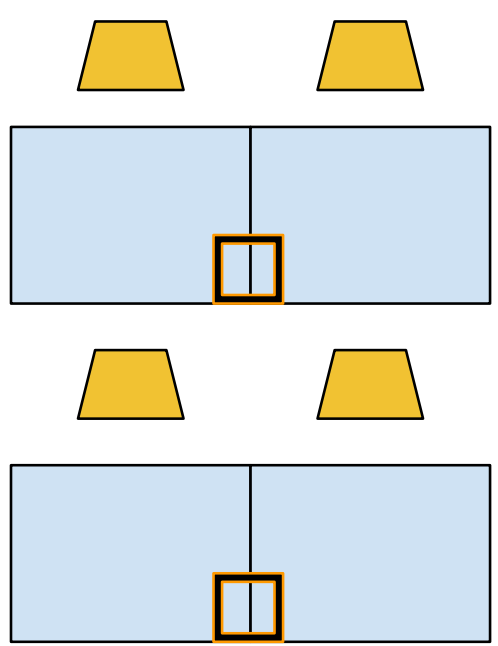
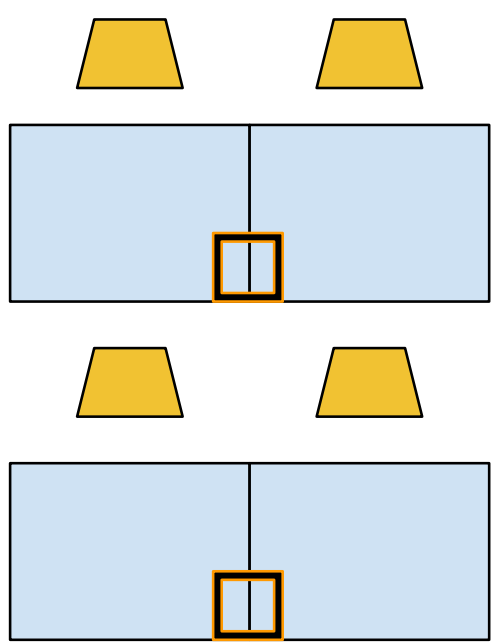

$\underline{\text { Key }}$

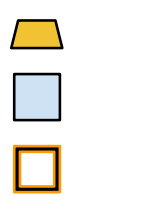

Chair Desk

FRONT

Figure 1. The visual of a small section of a classroom as seen below was used for the entirety of the testing for the experiment. 


\section{Results}

\section{Pre-survey Responses (From Control Group and Participants)}

All pre-survey data will be referred to the chart below, from both the control group and testing participants. As seen from both parties, the 19 out of 26 answered that they did not know what blue light therapy is, exposing how bluelight therapy is not well known and is still an invasive method. Regarding sleep, we can observe that since the testing group wakes up early for zero period, they receive less sleep, since they have the same workload as the control group but less time to manage. This is why none in the control group picked the option " $1-3$ hours" and picked more of the higher increments, also why some of the testing group did pick " $1-3$ hours" and no " $8-9$ hours." Furthermore, the control group did rate their quality of sleep higher than the testing group, which could also be from the difference in wake-up times. Expanding on morning fatigue, we can examine that few control group participants picked that they do not experience morning fatigue, compared to the testing group where none picked "no" and either picked "sometimes" or "yes." The final comparison is that the control group answered higher for the question about attentiveness in class. Overall, the pre-survey exhibits how my testing groups specific schedule compared to regular student's schedules differs and is much more diligent. This specific schedule would best display the effects of the blue-light therapy.

Table 1. Results from pre-survey were used as data to compare to results after testing

\begin{tabular}{|c|c|c|}
\hline Question & $\begin{array}{c}\text { Response (Average from all 16 } \\
\text { Participants/Testing Group) }\end{array}$ & $\begin{array}{c}\text { Response (From 10 Control Group } \\
\text { participants/Non-testing group) }\end{array}$ \\
\hline $\begin{array}{l}\text { Have you heard of Blue-light ther- } \\
\text { apy before or know what it is? }\end{array}$ & & \\
Yes & & \\
\hline $\begin{array}{l}\text { No } \\
\text { sleep do you get on weekdays? }\end{array}$ & & \\
\hline $\begin{array}{l}\text { 8-9 Hours } \\
\text { 6-7 Hours } \\
\text { 4-5 Hours }\end{array}$ & & \\
1-3 Hours & & \\
\hline
\end{tabular}




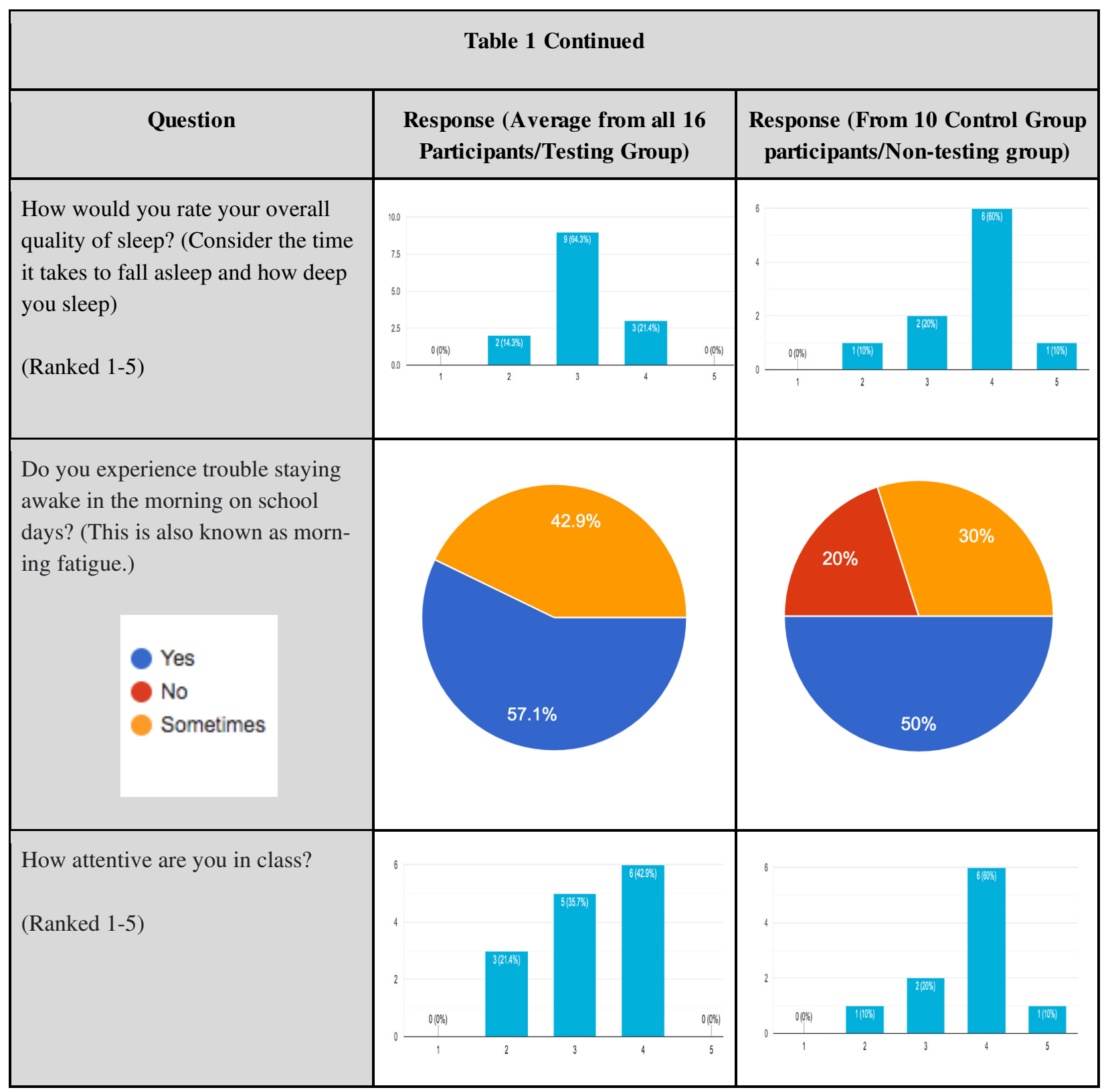

\section{Session One Results}

During the first session of testing, according to the results from the daily log that each participant filled out (both sessions data is charted below), there was a steady increase in attentiveness in class. The daily log asked questions regarding attentiveness on a rating scale of one through five, and as days passed, participants gradually ranked higher on the scale illustrating constant, steady use of the lightbox and promising improvement. Some stated that their work ethic was impacted greatly, in which they felt more motivated to get work completed and learn. For example, a student stated at the end of the two-week session:

"I felt instantly awake and refreshed after using the lightbox.. Throughout the rest of the day as well." - Anonymous Session One Participant 
This example depicts how one felt awake after usage of the lightbox in the morning and many others had similar reviews. Numerous participants became eager to come every day to the testing session as they were pleased with the results the lightbox delivered. The dose of blue light for about 15 minutes each morning allowed for a steady focus in school, enabling a better learning experience. Six out of eight participants from session one stated they would like to use the lightbox again with the results they have experienced.

\section{Session Two Results}

In close relation to session one results, session two answered and rated questions very similarly. Majority of the participants for this session, in the pre-survey, stated their average sleep on weekdays is 6-7 hours with few picking 1-3 and 4-5, and none picking 8-9 hours. After the two-week session, a significant amount picked a higher increment of sleep, meaning more picked 8-9 hours and fewer picking 1-3 and 4-5 hours. This evidently shows that the blue-light improved sleep significantly over the course of blue-light exposure. In regard to work ethic, students responded with positive results, as the majority of students picked 4-5 on a 1-5 scale rating attentiveness in class. In comparison to pre-testing where most students chose 1-3, signifying an apparent change. A student stated:

\section{"It lowered my anxiety and stress down"}

- Anonymous Session Two Participant

Elucidating how the blue-light helped one's mental state. This trend was prevalent in other participants as well. In comparison to pre-testing where most students chose 1-3, signifying an apparent change. Therefore, throughout session two, results proved the hypothesis to be true.

Throughout both testing sessions, the results proved my hypothesis to be correct. Over the course of four weeks, the two sessions produced results parallel to each other. In comparison to the pre-survey results, (all comparison following is in regard to the participants, not control group) there was an evident improvement in all elements I was testing for. To begin with, the participants during the pre-survey mostly said they slept 4-5 hours on weekdays, but compared to the daily log, more chose 8-9 hours and less 1-3 hours. Results on sleep quality displayed similar results, with the daily log results scoring better than the daily log. More students had chosen the highest ranking, five and fours, in comparison to the pre-survey where majority picked three, and no fives. Lastly, the ranking for attentiveness in class in the daily log also showed improvement alongside the other factors, with more participants picking higher rankings than the pre-survey. This demonstrates the stability and reliability of using the lightbox in order to improve work ethic and sleep cycle. 
Table 2. Daily Log Data (accumulative of both sessions)

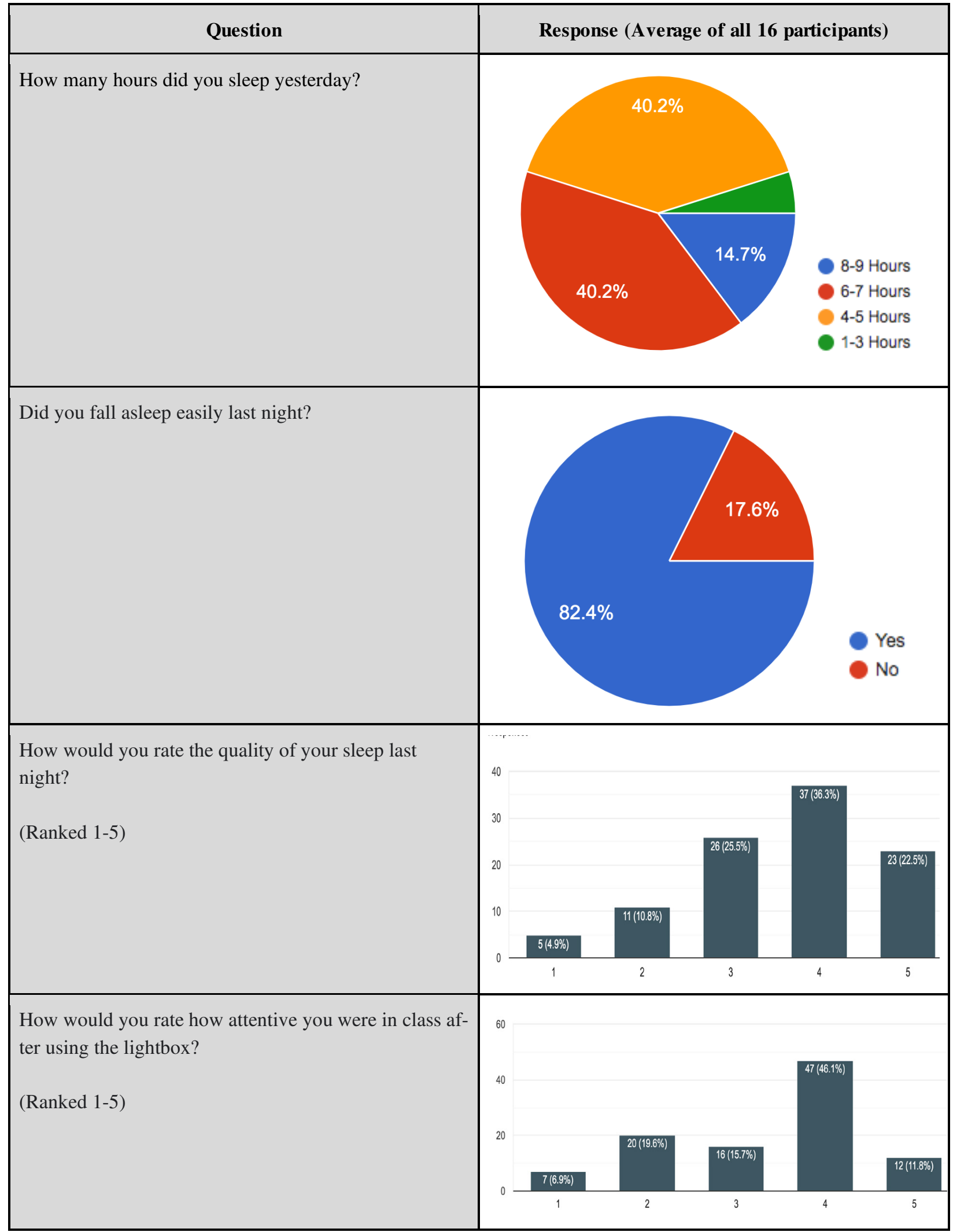




\section{Post-Survey Responses}

A post-survey was conducted at the end of both sessions to receive input about the overall participants' experience using the lightbox in comparison to the daily log. Questions were similar to the daily log but based on a more overall experience. This data is charted in Table 3.

Table 3. Post-survey input data about the overall participants' experience using the lightbox.

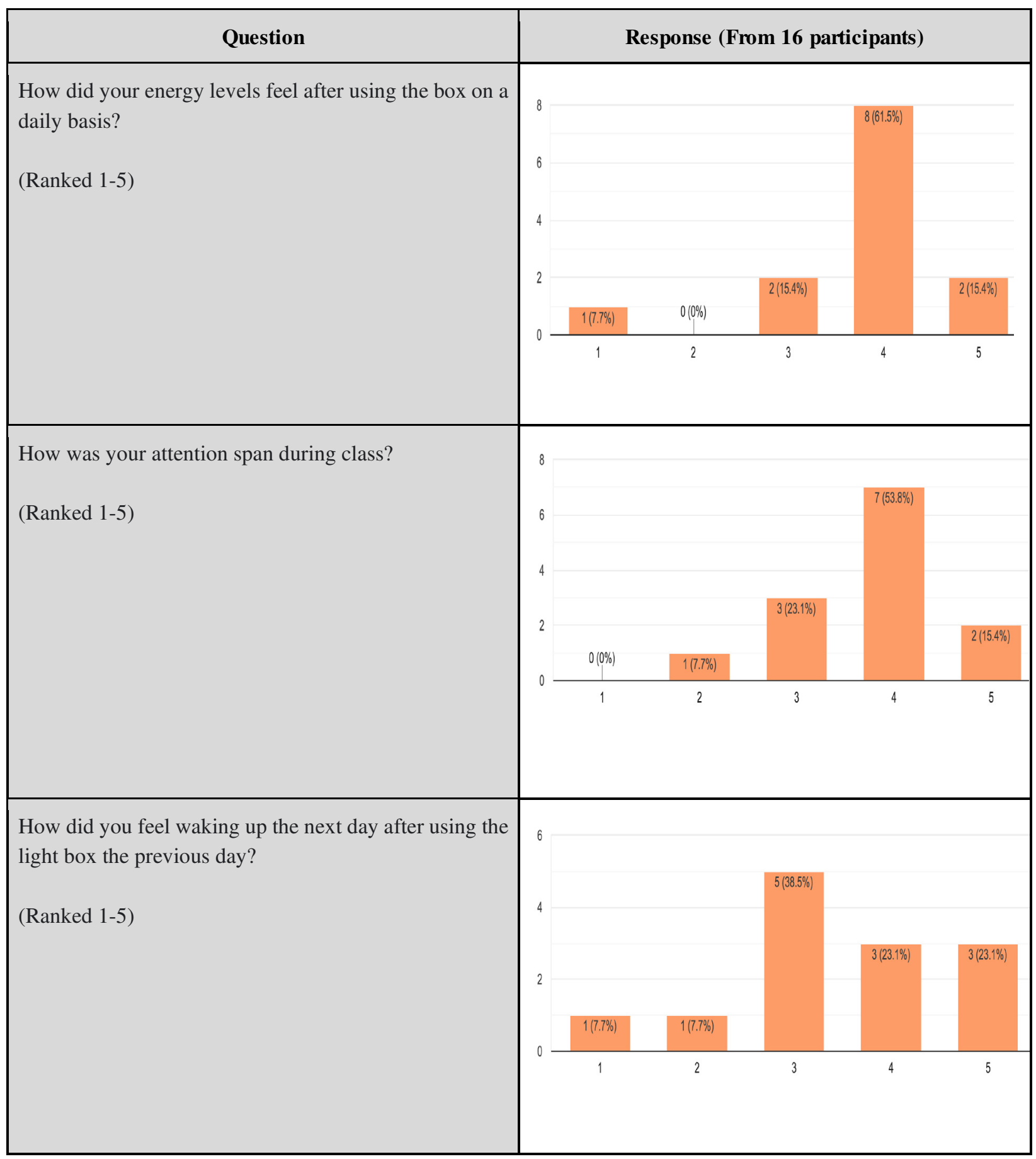




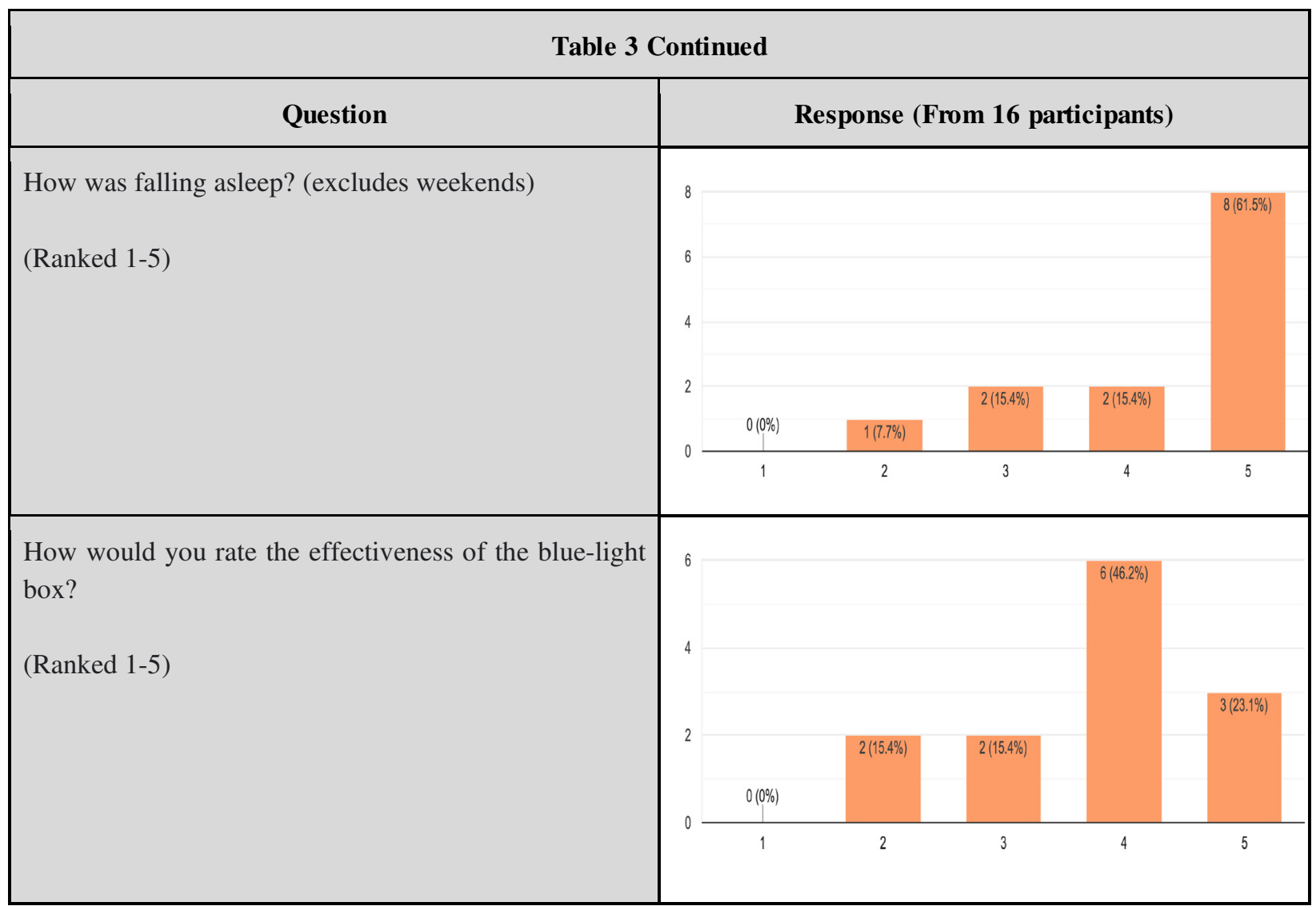

Overall, the post-survey responses provided the answer to my question. These results also proved my hypothesis to be true. Although some had rated the effectiveness of the lightbox poorly in some categories, this was due to personal reasons such as: not attending and not testing at the required amount of time, further explained in the limitations section. Aside from limitations, the lightbox tangibly improved the students' circadian rhythm where sleep was improved, even though there were short sleep times, there was deeper sleep within those limited hours. It was also effortless to fall asleep according to the high ranking fives from this survey. Along with the circadian rhythm, work ethic and attentiveness also increased overall, benefitting the participant.

\section{Discussion}

The data displayed throughout the experiment proved the hypothesis to be correct and valid through the use of various surveys. Using blue light in the form of lightboxes improves a high school student's attentiveness and work ethic, as well as including better quality sleep. The blue-light reacted with a participant's circadian rhythm and allowed a steady schedule to be followed, while being awake and allowing for attentive listening throughout class and a smooth slumber at night. In the future, blue-light therapy could be utilized by any ordinary student who struggles to stay awake in the morning and has trouble falling asleep at night. A student may use the box for 15-20 minutes in the mornings of school and allow their circadian rhythm to react beneficially. If this is implemented in schools and even office workplaces, such as a box somehow attached to a desk, it could enhance the ability to learn and accomplish work without feeling drowsy. This will improve the overall mood of the user, producing better outcomes, whether it be performance in school or work. If widespread in schools, blue-light therapy lamps could ensure effective study and attention, bettering education and possibly conduct. Through the results of this study, other researchers could use this data to support effective methods to improve performance in school. This data could be built upon and enhanced by testing other parts 
of the world, such as parts where sunlight is limited as in Finland or Norway. Any other special conditions applied could be experimented with as well. The future of improving student performance and sleep cycles possibly lies within blue-light therapy lightboxes.

\section{Limitations}

Throughout both sessions, some participants could not make it to testing on certain days due to personal matters, so for some using the blue-light box was nonconsecutive. Days absent from testing were restricted from going over two days (this was agreed to on the parental consent form), to keep data as accurate as possible. This affected my content in which the daily log was answered with a low rating of effectiveness sometimes, since the participant was absent on the previous day, therefore was not exposed to the blue-light. Also, some who did not sleep enough the previous day due to not sleeping at a certain time, answered low scaling during the daily log due to lack of energy for the blue-light to react with. This also explains why some had chosen the "1-3 hour or 4-5 hours" option in the daily log because they did not go to bed. Lastly, the final limit I encountered was that on a few days, testing time was cut down by a couple minutes, due to the testing site not opening on time as I could not access without the teacher present. Data overall was not largely impacted, but slightly due to these limitations.

\section{Implications}

Majority of participants said they would most likely use a lightbox again, after witnessing a dramatic change in work ethic and attention levels in school, followed by a better-night sleep. This illustrates how my findings connect to information collected in my literature review regarding blue-light. As blue light is seen to improve health in SAD patients according to Meesters, it also is effective for stressed high school students based on my research (2016). Furthermore, the students' circadian rhythm during the testing sessions became much healthier and stable, despite the early wake-up time for school. Based on my results, many felt refreshed in the morning even though sleep was limited and reported more energized and eager to learn in class. As the circadian rhythm in humans is sensitive to light, "we continually choose where to allocate attention, and what to ignore" explaining how humans are constantly impacted by everyday light (Collet et al. 2020). Therefore, the blue-light used during my research proved to improve students' attention spans and capacity to learn, controlling their circadian rhythm effectively.

\section{Conclusion}

Boxed Blue-Light Therapy proves to be effective in helping high-school students answering my question if "Does the use of blue-light therapy positively impact current high-school students in Southern California regarding morning fatigue and work ethic?" based on my research and results. Throughout the process, participants recorded positive results derived from using the lightbox during the assigned sessions and even continued usage after testing was completed because few wanted to continue with the benefits the light provided. If my research were to be expanded by future successors, I would optimistically increase the population and participant range to college students, even adults working a full-time job to see the effects. Overall, I am immensely pleased with the results I have received and the beneficial influence on students during the experiment.

\section{References}

Bagherani, N. (2016). Efficacy of blue light in treatment of acne. Dermatologic Therapy, 29(3), 210. https://doi.org/10.1111/dth.12291 
Collet, J., Ftouni, S., Clough, M., Cain, S. W., Fielding, J., \& Anderson, C. (2020). Differential Impact of Sleep Deprivation and Circadian Timing on Reflexive Versus Inhibitory Control of Attention. Scientific Reports, 10(1), 110. https://doi.org/10.1038/s41598-020-63144-y

Desan PH, Weinstein AJ, Michalak EE, et al. A controlled trial of the Litebook light-emitting diode (LED) light therapy device for treatment of Seasonal Affective Disorder (SAD). BMC Psychiatry. 2007;7:38-8. doi:10.1186/1471-244X-7-38.

Gold, M. H., Sensing, W., \& Biron, J. A. (2011). Clinical efficacy of home-use blue-light therapy for mild-to moderate acne. Journal of Cosmetic \& Laser Therapy, 13(6), 308-314. https://doi.org/10.3109/14764172.2011.630081

JACKSON, T. L., \& COOPER, T. (2020). Sleep Deprivation among Rhode Island High School Students. Rhode Island Medical Journal, 103(2), 49-52.

Lack, L., Bramwell, T., Wright, H., \& Kemp, K. (2007). Morning blue light can advance the melatonin rhythm in mild delayed sleep phase syndrome. Sleep \& Biological Rhythms, 5(1), 78-80. https://doi.org/10.1111/j.1479$\underline{8425.2006 .00250 . x}$

Loredana-Sabina P, Perri D, Vlad BA, Ciubara A, Marilena M, Virginia M. The Effects of Blue Light in Modern Society. BRAIN: Broad Research in Artificial Intelligence \& Neuroscience. 2019;10:5-11. http://search.ebscohost.com/login.aspx?direct=true \&db=asn\&AN=137195072\&site=ehost-live. Accessed January 6, 2020.

Meesters Y, Winthorst WH, Duijzer WB, Hommes V. The effects of low-intensity narrow-band blue-light treatment compared to bright white-light treatment in sub-syndromal seasonal affective disorder. BMC Psychiatry. 2016;16:110. doi:10.1186/s12888-016-0729-5.

Miura, J., Yuasa, T., Sugai, Y., Yamagami, K., \& Aizu, Y. (2018). Effects of Bright Light with Reduced Blue Light on Sleepiness on Rising: A Small Exploratory Study. Sleep Disorders, 1-7. https://doi.org/10.1155/2018/2378630

MIYAMOTO, M., MIYAMOTO, T., HIRATA, K., IWATA, K., NAKAJIMA, I., NARUKAWA, K., ... IMAI, Y. (2003). Unrefreshing sleep and daytime sleepiness contributing to complaints of morning headaches in sleep apnea syndrome patients. Sleep \& Biological Rhythms, 1(2), 117-119. https://doi.org/10.1046/j.1446-9235.2003.00016.x NCED. (2019). Back to School Statistics. Retrieved from https://nces.ed.gov/fastfacts/display.asp?id=372

Pradhan, R., \& Sinha, N. (2017). Impact of commuting distance and school timing on sleep of school students. Sleep \& Biological Rhythms, 15(2), 153-158. https://doi.org/10.1007/s41105-017-0091-0

Ranasinghe, A. N., Gayathri, R., \& Vishnu Priya, V. (2018). Awareness of effects of sleep deprivation among college students. Drug Invention Today, 10(9), 1806-1809.

Tien-Yi Tzung, Kuan-Hsing Wu, \& Mei-Lun Huang. (2004). Blue light phototherapy in the treatment of acne. Photodermatology, Photoimmunology \& Photomedicine, 20(5), 266-269. https://doi.org/10.1111/j.1600-

0781.2004.00109.x

Van Hoof, J., Westerlaken, A. C., Aarts, M. P. J., Wouters, E. J. M., Schoutens, A. M. C., Sinoo, M. M., \& Aries, M. B. C. (2012). Light therapy: Methodological issues from an engineering perspective. Technology \& Health Care, 20(1), 11-23. https://doi.org/10.3233/thc-2011-0650 